Brit. J. vener. Dis. (1958), 34, 219.

\title{
CHRONIC BENIGN CIRCUMSCRIBED PLASMA CELL BALANO-POSTHITIS*
}

\author{
BY \\ W. FOWLER \\ The Royal Hospital, Wolverhampton
}

Chronic benign circumscribed plasma cell balanoposthitis is the name given by Zoon (1952) to a disease which clinically resembles the erythroplasia described by Queyrat (1911) but has a distinctive histology.

Zoon (1952) studied the disease in eight patients; two of these were in the third decade of life, one was 58 years old, and the others 60 years or more. The duration of the disease was unknown in two cases, one year in two cases, and 12, 15, and 20 years in the remainder. In the majority of cases both the glans penis and the prepuce were affected. In one case the disease was limited to the inferior surface of the prepuce and in another to the area around the urinary meatus.

Histologically one case showed a moderate, benign acanthosis, and in the others the epidermis tended to be atrophied and was usually reduced to a few layers of cells. In some areas there was intra- and extra-cellular oedema. Some of the epidermal cells were vacuolated and resembled dyskeratotic cells. The granular layer was absent in every case and there was no hyperkeratosis. The dermal papillae were absent and the epidermo-dermal junction was a straight line. There was an inflammatory infiltration in the upper part of the corium, consisting of plasma cells with a few lymphocytes, eosinophils, and fibroblasts. There were many dilated capillaries, the walls of which were often thickened. The connective tissue was oedematous. The elastic tissue fibres stained normally. The prussian blue reaction was negative. Two patients received no treatment, one responded to contact $x$-ray therapy, and five resisted all treatment including radiotherapy.

Zoon (1952) was unable to discover the aetiology of the disease which was neither a toxic eruption, nor due to venereal disease, tuberculosis, or external irritation.

As chronic benign circumscribed plasma cell balano-posthitis does not appear to be very common, the following cases may be of interest.

\section{Case Reports}

Case 1, a widower aged 75 years, attended hospital on April 16, 1955, complaining of swelling and inflammation

\footnotetext{
* Received for publicatinn March 29, 1958.
}

of the penis of a few days' duration. Recently he had noticed that it was becoming increasingly difficult to retract the prepuce. There was no history of recent coitus and he had never suffered from venereal disease.

Examination.-The condition appeared to be a simple paraphimosis with secondary balano-posthitis.

Treatment.-The paraphimosis was reduced easily. The balano-posthitis responded slowly to treatment with simple antiseptics, and by May 16,1955, the prepuce was normal and the glans was also normal except for some brown staining on the right side of the dorsum. This staining disappeared during the next week and the patient was discharged as cured on June 25 . The prepuce was tight but could be retracted and circumcision was not indicated in view of his age.

Progress.-He remained well until December 31, 1955, when he felt some subpreputial irritation and noticed that the glans penis and prepuce were inflamed. He attended hospital on January 4, 1956.

On this occasion the greater part of the right side of the dorsum of the glans was occupied by a dry, shining, nonindurated, patchy, erythematous lesion which was well demarcated from the surrounding skin by intensely inflamed papules. There was a similar lesion on the contiguous surface of the prepuce and this extended into the coronal sulcus (Fig. 1). The inguinal glands were not enlarged and the skin and mucous membrane elsewhere were normal.

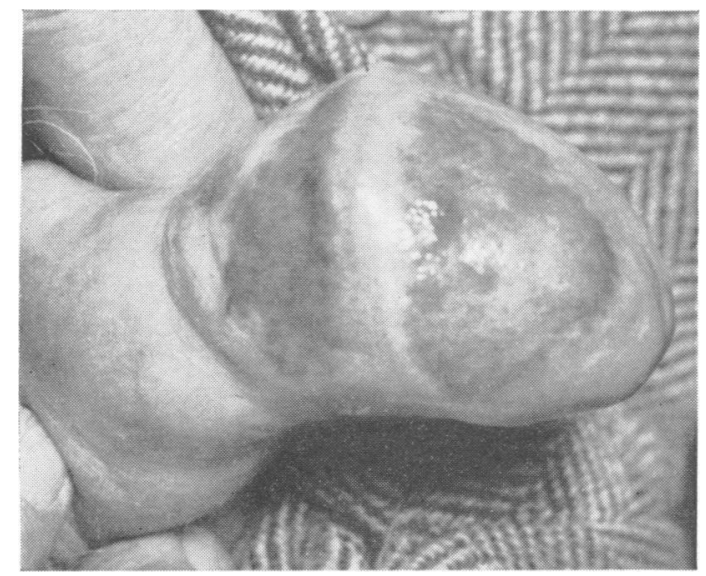

FIG. 1.-Chronic benign circumscribed plasma cell balano-posthitis. 
Arrangements for biopsy were made, but when the patient attended for this investigation the condition showed so much improvement that biopsy was postponed. Calamine liniment was prescribed and on February 11 the glans and prepuce again appeared normal.

The patient was next seen on January 7, 1957, when he complained of severe subpreputial irritation, and stated that during the previous 11 months there had been periodic attacks of slight balano-posthitis which disappeared spontaneously.

On examination the condition was essentially the same as on January 4th, 1956. Biopsy was carried out on January 10.

Histological Report.-There is some parakeratosis. The epidermal cells seem to be separated by intercellular oedema. The basal layer of the epidermis shows degeneration. The superficial corium is densely infiltrated with plasma cells. The infiltrate also contains a few polymorphs, lymphocytes, and tissue cells. The upper corium is extremely vascular but no free iron can be demonstrated, though Nodl (1954) obtained a positive prussian blue reaction in his case. The histological appearance is shown in Figs 2 and 3.

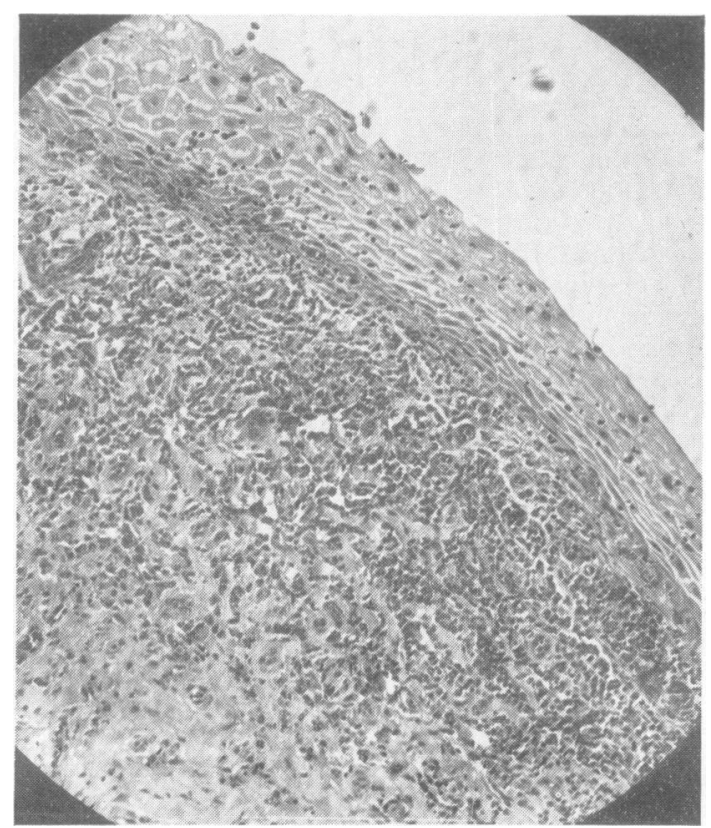

FIG. 2.-Inter-cellular oedema in epidermis, absence of papillae, and plasma cell infiltration in upper corium with increased vascularization. Haematoxylin and eosin $\times 84$ approx.

Progress.-After the biopsy the condition improved until by February 11, 1957, only the edge of the lesion was visible. Calamine liniment was the only treatment used at this time.

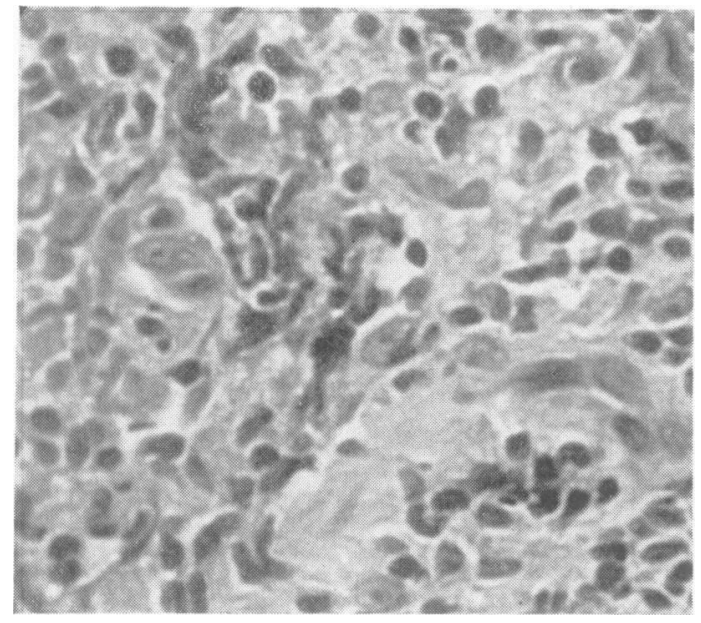

FIG. 3.-Cellular infiltration in Fig. 2. $\times 100$ approx.

The patient did not return to the clinic until August 22, 1957, when he stated that the balano-posthitis had never completely disappeared but that there had been no symptoms until a few days previously. The lesion appeared exactly the same as in the two previous years.

Calamine liniment had no influence on the lesion at this time nor had 1 per cent. hydrocortisone ointment, but when the strength of the latter ointment was increased to 2 per cent. hydrocortisone the symptoms were relieved and all but the edge of the lesion disappeared.

The patient is still under observation, the disease has shown no further tendency to spontaneous remission; 2 per cent. hydrocortisone ointment still relieves the symptoms, but is gradually losing the power to influence the appearance of the lesion.

Case 2, a 28-year-old Indian who had developed gonorrhoea 6 days previously, attended the clinic on March 13, 1957. There was no past history of venereal disease, and one injection of 300,000 units procaine penicillin cured the gonococcal infection, but on April 15, 1957, he complained of slight subpreputial irritation.

Examination.-A patch of balanitis occupied about two thirds of the dorsum of the glans. The affected area was well demarcated from the surrounding skin, being non-indurated, smooth, shining, and dark red in colour. The proximal edge of the lesion consisted of more intensely inflamed small papules (Fig. 4, opposite). The prepuce was not involved and the preputial ring was not narrowed, and the inguinal glands were not enlarged. The skin elsewhere was normal and there were no lesions in the mouth. Although no treatment was prescribed during the next 2 weeks the erythema gradually decreased until the lesion was barely visible. However, the condition soon returned to the original state and biopsy was carried out on May 27, 1957.

Histological Report.-There is parakeratosis and moderate acanthosis. Immediately under the epidermis 


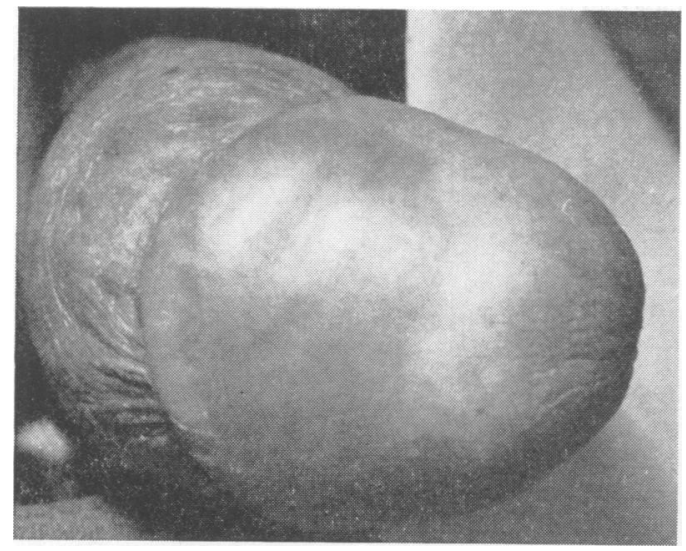

FIG. 4.-Chronic benign circumscribed plasma cell balanitis.

there is a heavy cellular infiltration, largely composed of plasma cells and also containing some polymorphs, lymphocytes, and tissue cells (Figs 5 and 6).

Progress.-The patient is still under observation. The disease has shown no further tendency to spontaneous remission, but has not extended. Antihistamine creams and various paints and antiseptic lotions have proved ineffective, but with hydrocortisone ointment the lesion disappears in 2 to 5 days. When this treatment was instituted 0.5 per cent. hydrocortisone was sufficient to produce this response, but 2 per cent. is now necessary.

\section{Comment}

The general health of both patients is excellent. There was no history of skin disease before the present illness nor of the habitual taking of medicaments. The urine has been normal throughout the course of the disease and serum tests for syphilis have been persistently negative. Neither the red nor the white blood cells have shown any abnormality and the serum fibrinogen has remained within normal limits. The total serum protein has varied from 6.0 to $7.5 \mathrm{~g}$. per $100 \mathrm{ml}$., and the serum globulin from 1 to $1.8 \mathrm{~g}$. per $100 \mathrm{ml}$. Paper electrophoresis suggested that the alpha-2 globulin was slightly increased and the gamma globulin slightly decreased.

The diagnosis in Case 2 might be questioned, as clinically this case differs from Case 1 and histologically the epidermal changes differ both from those of Case 1 and from those of seven of the cases studied by Zoon (1952). However acanthosis was a feature of one of Zoon's cases, and it is so unusual to find a plasma cell infiltration in simple balanitis that the diagnosis is not considered to be in any doubt.

Case 1 shows that at times spontaneous remissions are a feature of the early stages of the disease. This

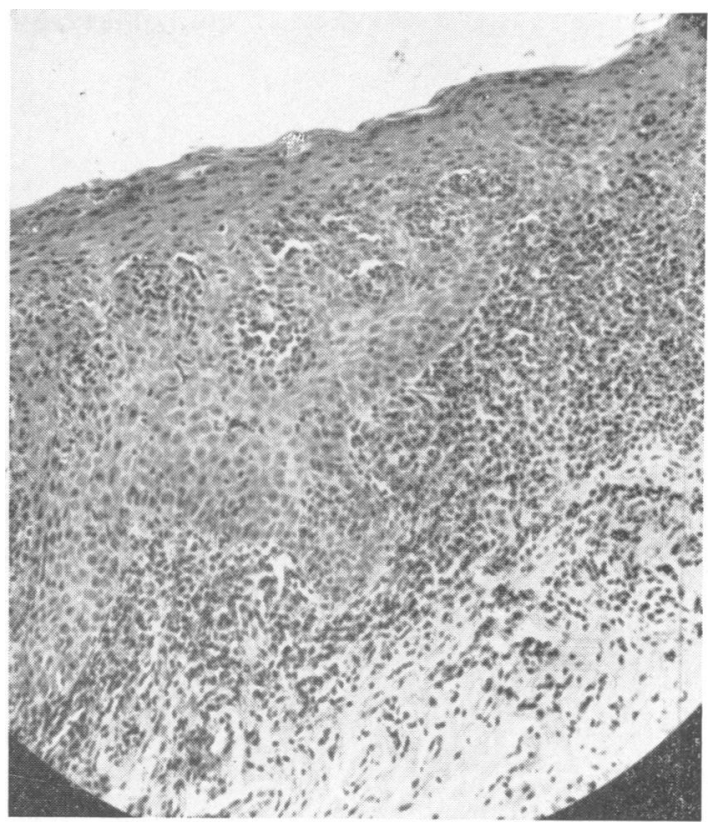

FIG. 5.-Parakeratosis, acanthosis, and dense cellular infiltration immediately under the epidermis. Haematoxylin and eosin $\times 90$ approx.

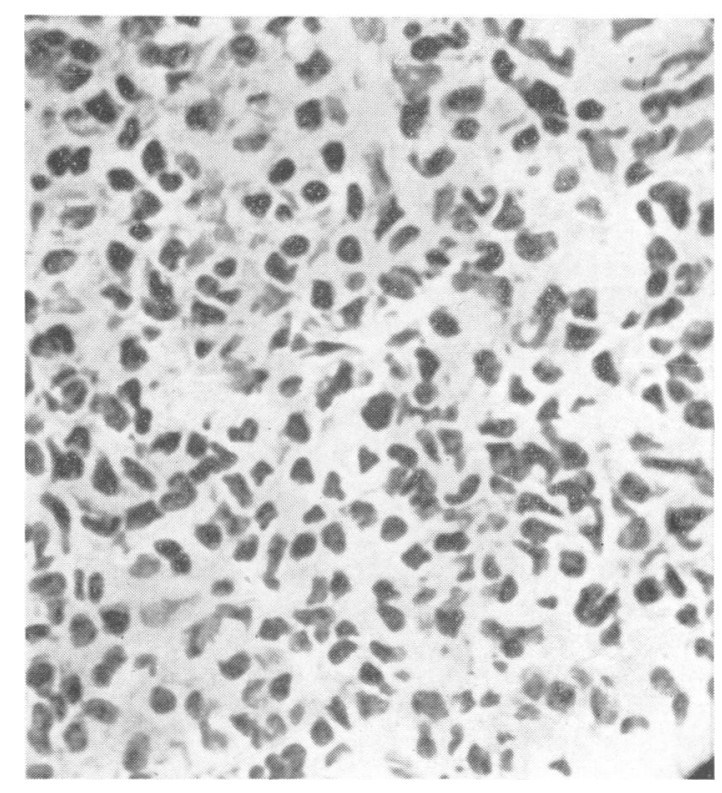

Fig. 6.-Infiltration in Fig. 5, showing preponderance of plasma cells $\times 160$ approx.

and the diminishing response shown to hydrocortisone by both cases suggest that the disease process becomes more intense with the passage of time. 


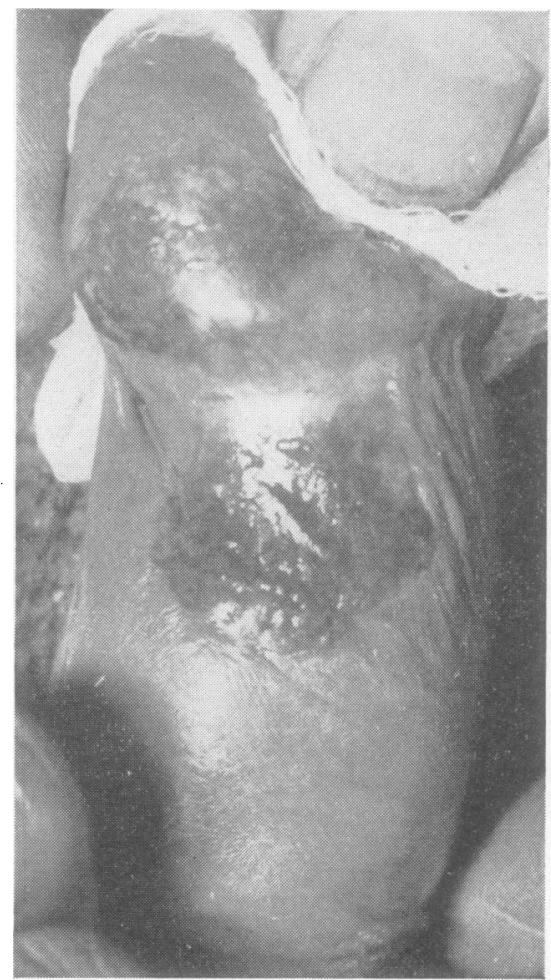

FIG. 7.-Chronic non-specific balano-posthitis.

Zoon (1952) stressed that chronic benign circumscribed plasma cell balano-posthitis was clinically indistinguishable from the erythroplasia of Queyrat, and under the latter heading he includes malignant as well as pre-malignant conditions. It may also be difficult to differentiate on clinical grounds, between chronic benign circumscribed plasma cell balano-posthitis and simple balanitis.

The condition shown in Figs 7 and 8 illustrates this point. This patient was 46 years of age, and no cause was found for the balano-posthitis which persisted for over 2 months. The histological changes were non-specific and reminiscent of eczema.

The fact that the disease followed an attack of gonorrhoea in Case 2 is interesting, but of no aetiological significance. It is tempting to attribute some responsibility for the disease in Case 1 to the partial phimosis. However, only one of Zoon's

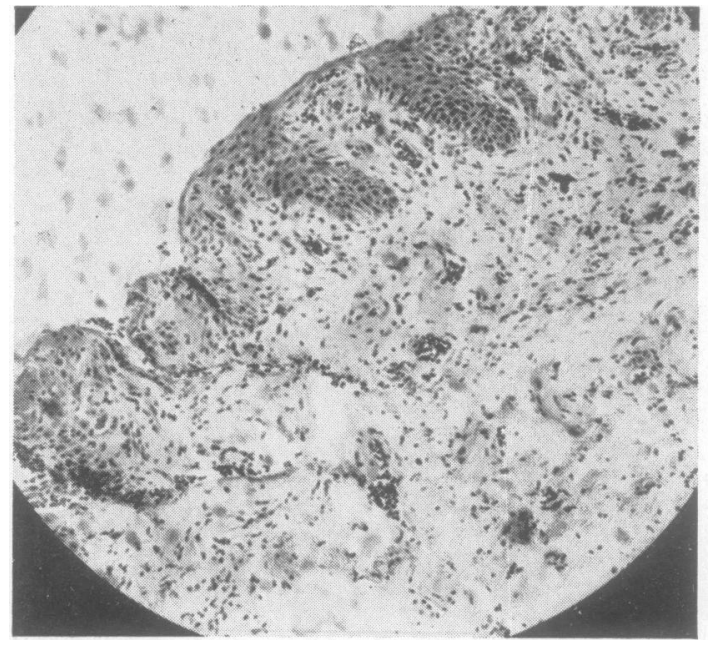

FIG. 8.-Thinning of epidermis over papillae, thickening of rete pegs, mild inter-cellular oedema in epidermis, increased vascularization in corium, and light infiltration with lymphocytes, plasma cells, and mast cells.

patients had a tight prepuce and according to this author chronic benign circumscribed plasma cell balano-posthitis has been recorded in Jews under the heading of the erythroplasia of Queyrat.

My cases add nothing to the knowledge of the aetiology of the disease, and no positive contribution has been made to the therapeutics of chronic benign circumscribed plasma cell balano-posthitis except to show that hydrocortisone can cause the symptoms and signs to disappear temporarily.

\section{Summary}

The work of Zoon (1952) on chronic benign circumscribed plasma cell balano-posthitis is briefly reviewed, and two cases are recorded.

I am grateful to Dr. A. G. Marshall, M.D., senior pathologist to the Royal Hospital, for the biopsy reports and to Mr. D. R. Paton, medical photographer to the hospital, for the plates.

\section{REFERENCES}

Nödl, F. V. (1954). Arch. Derm. Syph. (Berl.). 198, 557.

Queyrat, L. (1911). Bull. Soc. franc. Derm. Syph., $22,378$. Zoon, J. J. (1952). Dermatologica (Basel), 105, 1. 